\title{
Cavernomas da fossa posterior do crânio - Relato de série de seis casos
}

\author{
Rodrigo Moreira Faleiro', Luanna Rocha Vieira Martins²
}

Hospital Felício Rocho, Belo Horizonte, MG, Brasil e Universidade Federal de Minas Gerais, Belo Horizonte, MG, Brasil.

\section{RESUMO}

Os cavernomas são lesões classificadas como malformações vasculares, juntamente com malformações arteriovenosas, desenvolvimento venoso anômalo e telangiectasias capilares. Podem ocorrer de forma esporádica ou familiar e acometer qualquer área do sistema nervoso central, sendo a região supratentorial sua localização mais frequente. Neste artigo, é descrita uma série de seis pacientes com cavernoma na fossa posterior. Cinco cavernomas localizavam-se no tronco encefálico e um no cerebelo. A faixa etária dos pacientes variou de 14 a 50 anos. A abordagem cirúrgica diferiu entre os casos. Quatro casos apresentaram piora no pós-operatório imediato, com melhora subsequente. O seguimento dos pacientes variou de quatro meses a sete anos. Não houve óbito na série. Os cavernomas da fossa posterior requerem abordagem individualizada e técnica cirúrgica acurada. Embora possa ocorrer piora momentânea no pós-operatório imediato, a melhora subsequente é a regra, sendo o óbito infrequente.

\section{PALAVRAS-CHAVE}

Malformações vasculares do sistema nervoso central, fossa craniana posterior, hemangioma cavernoso.

\section{ABSTRACT}

Posterior fossae cavernomas - Study series of six cases

Cavernomas are lesions classified as vascular malformations, along with arteriovenous malformations, developmental venous anomalies and capillary telangiectasia. They can occur in a sporadic or familial form and can affect any area of the central nervous system, being the region supratentorial the most frequent location. In this article, we describe a series of six cases of posterior fossae cavernoma. Five cavernomas were located in the brainstem and one in cerebellum. The ages ranged from 14 to 50 years old. The surgical approach differed between cases. Four cases worsened in the immediate postoperative period, with subsequent improvement. The follow-up ranged from four months to seven years. There were no deaths. Posterior fossae cavernomas require individualized approach and accurate surgical technique. Although momentary worsening may occur in the immediate postoperative period, the subsequent improvement is the rule, and the death is infrequent.

\section{KEYWORDS}

Central nervous system vascular malformations, cranial fossa posterior, hemangioma cavernous.

1 Chefe do Serviço de Neurocirurgia e Neurologia do Hospital João XXIII e neurocirurgião do Hospital Felício Rocho, Belo Horizonte, MG, Brasil.

2 Acadêmica de Medicina da Universidade Federal de Minas Gerais (UFMG), Belo Horizonte, MG, Brasil. 


\section{Introdução}

Os cavernomas são lesões classificadas como malformações cerebrovasculares juntamente com as malformações arteriovenosas, desenvolvimento venoso anômalo e telangiectasias capilares. Representam a segunda malformação cerebrovascular mais frequente, constituindo $10-15 \%$ do total. ${ }^{1,2}$ A prevalência estimada na população geral foi de $0,4 \%$, segundo estudos baseados em autópsia e ressonância nuclear magnética. ${ }^{3}$

Os cavernomas são historicamente considerados lesões congênitas, vigentes em qualquer faixa etária, desde neonatos até adultos. No entanto, em alguns casos, podem estar associados com um histórico prévio de irradiação craniana ou infecção viral em indivíduos geneticamente predispostos. Ocorrem de forma esporádica ou familiar. ${ }^{4}$

Essas lesões podem ser encontradas em qualquer localização do sistema nervoso central, sendo a maioria localizada na região supratentorial. Dessa forma, os pacientes apresentam manifestações clínicas diversas como convulsões (23-50\%), cefaleia (6-52\%) e déficits focais (20-45\%) decorrentes principalmente de hemorragia, mas também de efeito de massa ou depósito de hemossiderina perilesional; no entanto, mais de $40 \%$ dos pacientes permanecem assintomáticos. ${ }^{3,5}$

Cavernomas da fossa posterior são menos frequentes e requerem conduta individualizada e técnica cirúrgica acurada. Em geral, esses casos têm bom prognóstico, sendo o óbito infrequente.

A seguir, é descrita uma série de seis casos de cavernoma da fossa posterior.

\section{Relato de casos}

Trata-se de estudo retrospectivo e descrição de série de casos. Foram analisados seis casos de cavernoma da fossa posterior.
Em relação à localização anatômica, cinco cavernomas localizavam-se no tronco encefálico, sendo dois no mesencéfalo, um na ponte, um na região pontomesencefálica e um na região bulbopontina. Outro paciente apresentava cavernoma no hemisfério cerebelar direito. Ao diagnóstico, os pacientes apresentavam manifestações clínicas diversas.

Quanto ao perfil dos pacientes analisados, quatro eram do sexo feminino e dois do masculino, com faixa etária variando de 14 a 50 anos. O seguimento desses pacientes variou de quatro meses a sete anos (Tabela 1).

Os acessos cirúrgicos utilizados para a abordagem dos cavernomas do tronco encefálico foram: fronto-orbitozigomáticos em dois casos, retrossigmóideo em dois casos e suboccipital mediano em um caso. O cavernoma cerebelar teve sua abordagem por via suboccipital lateral. Conseguiu-se exérese completa em cinco casos e parcial em um caso. Dois pacientes não apresentaram nenhuma piora no pós-operatório imediato e quatro pacientes apresentaram piora discreta, mas com impressionante recuperação nas primeiras semanas de pós-operatório. Não houve óbito na presente série (Figura 1).

\section{Discussão}

Os cavernomas são lesões circunscritas, multilobuladas, com aspecto que se assemelha, segundo alguns autores, à amora., ${ }^{2,6}$ Quando localizados intracranialmente, encontram-se frequentemente na região supratentorial (64-84\%), sendo localizados preferencialmente nos lobos frontal e temporal. Com menor frequência, acometem a região infratentorial (19-35\%), em que cerebelo e ponte são mais acometidos. Lesões espinhais, por sua vez, são localizadas principalmente na região toracolombar baixa (97\%). ${ }^{2,8}$ São observados em regiões corticais, bem como em regiões profundas, como gânglios da base, tálamo, cerebelo e tronco encefálico. ${ }^{9}$

\begin{tabular}{|c|c|c|c|c|c|}
\hline Paciente & Sexo & Idade & $\begin{array}{l}\text { Localização do } \\
\text { cavernoma }\end{array}$ & Apresentação clínica & Abordagem cirúrgica \\
\hline 1 & Feminino & 14 & Mesencéfalo & Hemiplegia D + Oftalmoplegia $\mathrm{E}$ & Via fronto-orbitozigomática \\
\hline 2 & Masculino & 19 & Pontomesencéfalo & $\begin{array}{l}\text { Hemiplegia + diplopia (abducente } \\
\text { bilateral) + paralisia facial periférica }\end{array}$ & Via suboccipital mediana \\
\hline 3 & Feminino & 17 & Ponte & $\begin{array}{l}\text { Dor e hipoestesia em hemiface D, } \\
\text { seguida de paralisia facial } \\
\text { periférica D + cefaleia }\end{array}$ & Via retrossigmóidea \\
\hline 4 & Masculino & 40 & Cerebelo & Cefaleia & Via suboccipital lateral \\
\hline 5 & Feminino & 36 & Bulbopontino & $\begin{array}{l}\text { Cefaleia e diplopia, seguida de } \\
\text { hemiparesia } \mathrm{E}+\text { paralisia facial central D }\end{array}$ & Via retrossigmóidea \\
\hline 6 & Feminino & 50 & Mesencéfalo & Oftalmoplegia D + hemiparesia $\mathrm{E}$ & Via fronto-orbitozigomática \\
\hline
\end{tabular}


Os achados histológicos dos cavernomas compreendem vasos sanguíneos dilatados com paredes espessas e amorfas - cavernas, presença de trombos no interior

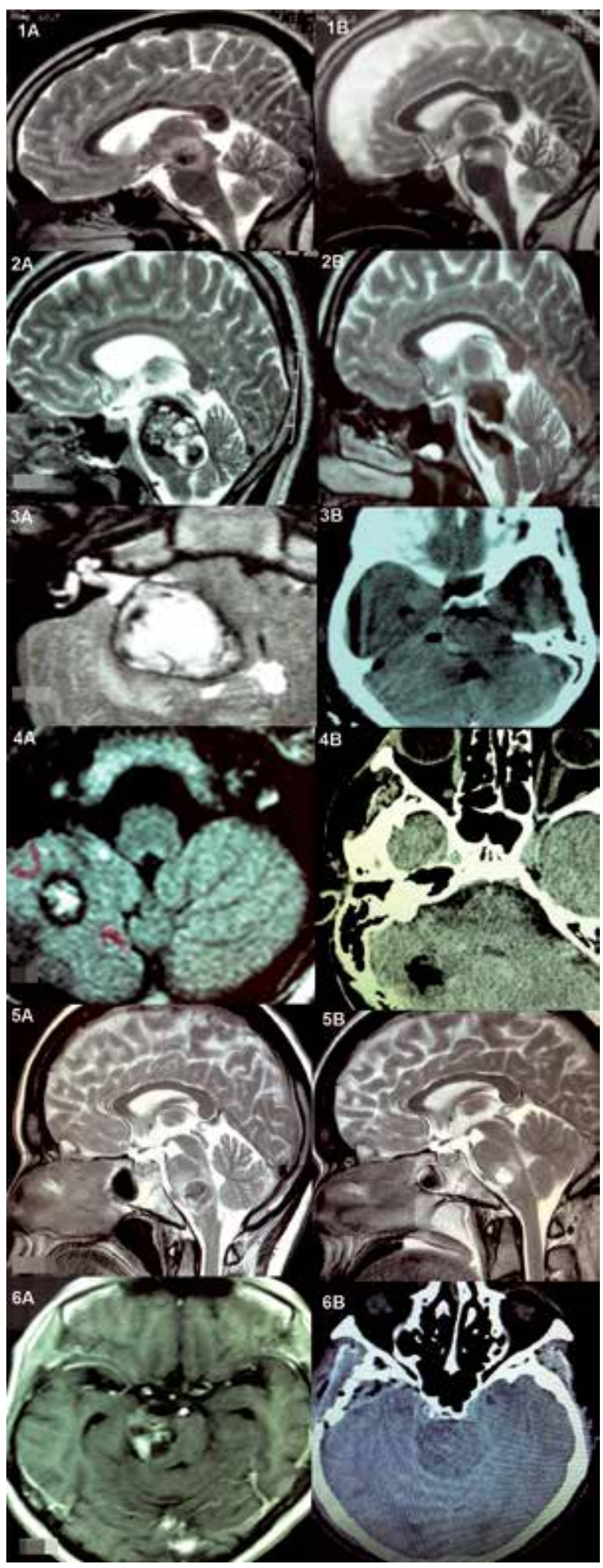

Figura 1 - Imagens do pré-e pós-operatório dos seis casos da série. $1 A, 2 A, 3 A, 4 A, 5 A, 6 A$ : pré-operatório. $1 B, 2 B, 3 B, 4 B, 5 B$, 6B: pós-operatório. dos vasos e fibrose com depósitos de hemossiderina circundando a lesão. ${ }^{10}$

A história natural dos cavernomas vem sendo investigada ao longo dos últimos 50 anos; contudo, ainda é difícil descrevê-la com clareza devido à falta de comparabilidade entre os estudos na literatura. Muitos estudos mostram um curso relativamente benigno, no que se refere à ocorrência de hemorragias, das lesões superficiais em comparação com as lesões profundas ou localizadas infratentorialmente. ${ }^{2,3,5}$ Segundo Kupersmith et al., ${ }^{11}$ hemorragias são mais frequentes em lesões associadas com desenvolvimento venoso anômalo, lesões sintomáticas em pacientes com idade inferior a 35 anos e em lesões com diâmetro maior que $1 \mathrm{~cm}$. Lesões assintomáticas tendem a seguir um curso mais benigno em relação às lesões sintomáticas, mesmo quando localizadas em regiões profundas. ${ }^{12}$ Pacientes com história de hemorragias ou déficits focais possuem maior risco de hemorragia subsequente. ${ }^{13}$ Cavernomas têm sido relatados em ambos os extremos da vida, embora a idade de início dos sintomas seja em torno de 35 anos de idade. ${ }^{14}$ Em geral, o prognóstico de pacientes mais jovens é pior em relação aos pacientes mais velhos, estando mais propensos à ocorrência de hemorragia e de déficits focais. ${ }^{2}$ Homens possuem maior probabilidade em apresentar sintomas em idade mais jovem e em ter convulsões; mulheres, por sua vez, têm o início dos sintomas em idade mais tardia e possuem maior probabilidade em ter déficits neurológicos. ${ }^{15}$ Essa discrepância entre os sexos tem sido atribuída às diferenças hormonais; o estrógeno possui possível efeito na angiogênese e na integridade estrutural da lesão, cuja ação é observada principalmente na gravidez. ${ }^{16}$

Os cavernomas geralmente não são detectados em angiografias em decorrência do fluxo sanguíneo muito lento ou da ocorrência de trombose espontânea. ${ }^{6}$ No entanto, eles são mais bem avaliados pela ressonância nuclear magnética (RNM), que mostra aspectos característicos da lesão. Tomografia computadorizada (TC) possui menor sensibilidade que a RNM, especialmente em lesões menores que $1 \mathrm{~cm},{ }^{17}$ sendo mais usada para avaliação de complicações como hemorragia aguda, para o seguimento de pacientes com diagnóstico prévio de cavernoma e para avaliação de estruturas ósseas da base do crânio da fossa posterior (grandes lesões podem causar remodelamento de ossos). ${ }^{18}$ RNM é o exame de imagem mais sensível e específico para o diagnóstico de cavernomas cerebrais. ${ }^{19}$

A conduta diante do diagnóstico de cavernoma ainda é controversa em decorrência da ausência na literatura de dados provenientes de grandes ensaios randomizados. Estudos disponíveis sugerem conduta expectante para pacientes com múltiplas lesões ou com lesões inacessíveis do ponto de vista cirúrgico. Conduta cirúrgica precoce, por sua vez, é indicada para pacientes com lesões únicas, acessíveis cirurgicamente e, sobretudo, 
sintomáticos. Pacientes jovens, com lesões acessíveis e ausência de comorbidades são os melhores candidatos para a ressecção. De forma geral, a decisão entre conduta expectante e cirúrgica deve balancear os riscos cirúrgicos e a probabilidade de a lesão se tornar sintomática. Além disso, deve-se considerar a carga psicológica imposta ao paciente, uma vez que ele passa a conviver com o conhecimento da presença de uma lesão que pode se tornar sintomática e gerar problemas súbitos. ${ }^{2}$

Os cavernomas cerebelares se comportam de forma análoga aos cavernomas supratentoriais. Sua abordagem cirúrgica deve envolver ressecção completa da lesão e do halo de hemossiderina. As lesões do tronco encefálico, por sua vez, possuem abordagem diferente e seguimos o proposto por Samii et al..$^{20}$ (Figura 2).
A literatura, assim como a presente série descrita, mostra que pacientes com cavernoma localizado na fossa posterior, em especial no tronco encefálico, requerem avaliação clínica e neurorradiológica cuidadosa e individualizada, especialmente quando a lesão está associada à hemorragia clinicamente significativa. Terapia curativa com microcirurgia possui, como visto na série, excelentes resultados. Embora possa ocorrer piora momentânea no pós-operatório imediato, a melhora subsequente é quase a regra. Modernos métodos diagnósticos e terapêuticos como monitoramento eletrofisiológico, neuronavegação e técnicas microcirúrgicas precisas, incluindo abordagem e respeito às zonas de entrada para o tronco encefálico, garantem o sucesso do procedimento cirúrgico. ${ }^{21}$

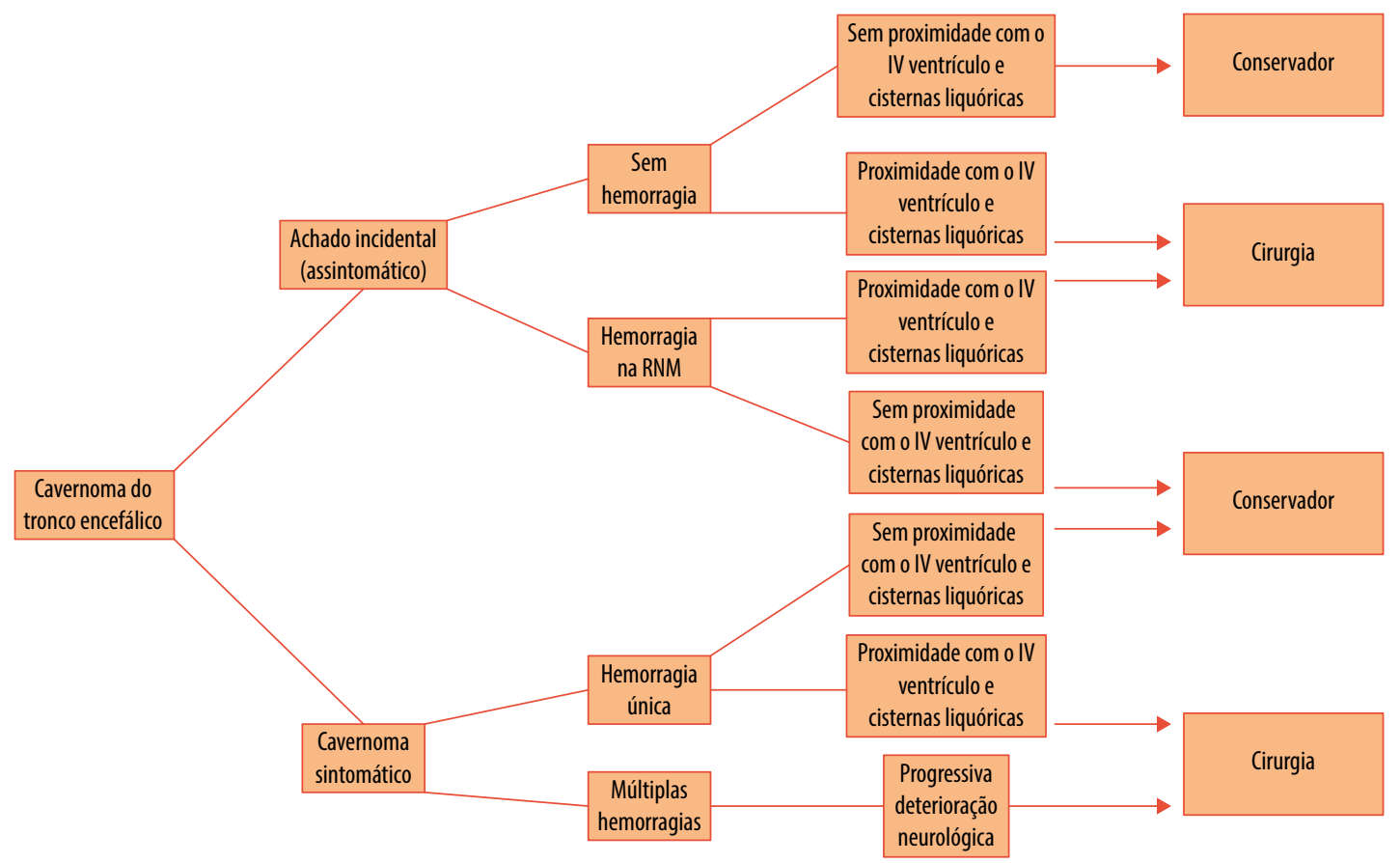

Figura 2 - Algoritmo, segundo Samii et al. ${ }^{20}$, para manejo dos cavernomas do tronco encefálico.

\section{Conflitos de interesse}

Os autores declaram não haver conflito de interesse.

\section{Referências}

1. Moriarity JL, Clatterbuck RE, Rigamonti D. The natural history of cavernous malformations. Neurosurg Clin N Am. 1999;10(3):411-7.

2. Maraire JN, Awad IA. Intracranial cavernous malformations: lesion behavior and management strategies. Neurosurgery. 1995;37(4):591-605.
3. Robinson JR, Awad IA, Little JR. Natural history of the cavernous angioma. J Neurosurg. 1991;75(5):709-14.

4. Pozzati E, Acciarri N, Tognetti F, Marliani F, Giangaspero F. Growth, subsequent bleeding, and de novo appearance of cerebral cavernous angiomas. Neurosurgery. 1996;38(4):662-9.

5. Porter PJ, Willinsky RA, Harper W, Wallace MC. Cerebral cavernous malformations: natural history and prognosis after clinical deterioration with or without hemorrhage. J Neurosurg. 1997;87(2):190-7.

6. McCormick WF, Hardman JM, Boulter TR. Vascular malformations ("angiomas") of the brain, with special reference to those occurring in the posterior fossa. J Neurosurg. 1968;28(3):241-51.

7. McCormick WF, Nofzinger JD. "Cryptic" vascular malformations of the central nervous system. J Neurosurg. 1966;24(5):865-75. 
8. Nozaki K, Inomoto T, Takagi Y, Hashimoto N. Spinal intradural extramedullary cavernous angioma. Case report. J Neurosurg. 2003;99(Suppl 3):316-9.

9. Simard JM, Garcia-Bengochea F, Ballinger WE Jr, Mickle JP, Quisling RG. Cavernous angioma: a review of 126 collected and 12 new clinical cases. Neurosurgery. 1986;18(2):162-72.

10. Tomlinson FH, Houser OW, Scheithauer BW, Sundt TM Jr, Okazaki H, Parisi JE. Angiographically occult vascular malformations: a correlative study of features on magnetic resonance imaging and histological examination. Neurosurgery. 1994;34(5):792-9.

11. Kupersmith MJ, Kalish H, Epstein F, Yu G, Berenstein $\mathrm{A}$, Woo $\mathrm{H}$, et al. Natural history of brainstem cavernous malformations. Neurosurgery. 2001;48(1):47-53.

12. Mathiesen T, Edner G, Kihlström L. Deep and brainstem cavernomas: a consecutive 8-year series. J Neurosurg. 2003;99(1):31-7.

13. Kondziolka D, Lunsford LD, Kestle JR. The natural history of cerebral cavernous malformations. J Neurosurg. 1995;83(5):820-4.

14. Del Curling O Jr, Kelly DL Jr, Elster AD, Craven TE. An analysis of the natural history of cavernous angiomas. J Neurosurg. 1991;75(5):702-8.

15. Casazza M, Broggi G, Franzini A, Avanzini G, Spreafico R, Bracchi $M$, et al. Supratentorial cavernous angiomas and epileptic seizures: preoperative course and postoperative outcome. Neurosurgery. 1996;39(1):26-32.

16. Aiba T, Tanaka R, Koike T, Kameyama S, Takeda N, Komata T. Natural history of intracranial cavernous malformations. J Neurosurg. 1995;83(1):56-9.

17. Kaard HP, Khangure MS, Waring P. Extraaxial parasellar cavernous hemangioma. AJNR Am J Neuroradiol. 1990;11(6):1259-61.

18. Lim DD, Wael A. Neuroimaging of cavernous malformations In: Rigamonti D, editor. Cavernous malformations of the nervous system. New York: Cambridge; 2011. p. 49-63.

19. Bradac GB, Riva A, Schörner W, Stura G. Cavernous sinus meningiomas: an MRI study. Neuroradiology. 1987;29(6):578-81.

20. Samii M, Eghbal R, Carvalho GA, Matthies C. Surgical management of brainstem cavernomas. J Neurosurg. 200;95(5):825-32.

21. Rigamonti D, editor. Cavernous malformations of the nervous system. New York: Cambridge; 2011.

\section{Endereço para correspondência}

Rodrigo Moreira Faleiro

Rua Caraça, 518, ap. 201, Serra

30220-260 - Belo Horizonte, MG, Brasil

E-mail: r.m.faleiro@hotmail.com 